\title{
INTEGRAL insight into the inner parts of the Galaxy. High mass X-ray binaries
}

\author{
A. Lutovinov ${ }^{1,2}$, M. Revnivtsev ${ }^{2,1}$, M. Gilfanov ${ }^{2,1}$, P. Shtykovskiy ${ }^{1,2}$, S. Molkov ${ }^{1,2}$, and R. Sunyaev ${ }^{1,2}$ \\ ${ }^{1}$ Space Research Institute, Russian Academy of Sciences, Profsoyuznaya 84/32, 117810 Moscow, Russia \\ e-mail: lutovinov@hea.iki.rssi.ru \\ ${ }^{2}$ Max-Planck-Institute für Astrophysik, Karl-Schwarzschild-Str. 1, 85740 Garching bei München, Germany
}

Received 18 November 2004 / Accepted 4 August 2005

\section{ABSTRACT}

Since its launch INTEGRAL has devoted a significant fraction of its observing time to the Galactic plane region. We present the results of INTEGRAL observations of the inner spiral arms of the Galaxy (Norma, Scutum and Sagittarius) with attention to high mass X-ray binaries. The increased sensitivity of the survey and possibility to detect absorbed sources significantly enlarged our sample of HMXBs in comparison with previous studies. For some absorbed sources we present for the first time high quality broadband (1-100 keV) energy spectra. We confirm pulsations of the X-ray flux of IGR J16358-4726, discovered by CHANDRA, and report discovery of pulsations with a period of $228 \pm 6 \mathrm{~s}$ from IGR J16465-4507. We confirm that the Galactic high mass X-ray binary distribution is significantly different from the distribution of low mass $\mathrm{X}$-ray binaries concentrated in the Galactic Center. A large fraction of detected high mass X-ray binaries are absorbed sources.

Key words. Galaxy: structure - binaries: general - X-rays: binaries

\section{Introduction}

One of the most interesting results obtained by the INTEGRAL observatory is the discovery of a large population of highly photoabsorbed Galactic X-ray sources. The first source discovered with INTEGRAL (IGR J16318-4848, Courvoisier et al. 2003) was shown to have a large intrinsic absorption in the X-ray spectrum (e.g. Murakami et al. 2003; de Plaa et al. 2003; Matt \& Guainazzi 2003; Revnivtsev et al. 2003a). After this source several more were discovered (e.g. IGR J16320-4751 = AX J1631.9-4752, IGR J16358-4726, etc.) which also have high photoabsorption, strongly exceeding that of the interstellar medium. It was proposed that these sources can be members of a previously poorly known population of Galactic X-ray sources with high intrinsic absorption (Revnivtsev et al. 2003a). Based on the photometric observations of the source IGR J16318-4848 these authors also suggested that the source could be a binary with a giant or supergiant companion with a strong stellar wind, that was later confirmed by precise spectroscopic observations of the optical counterpart (Filliatre \& Chaty 2004).

The analysis of properties of newly discovered absorbed sources showed that they are likely high mass X-ray binaries (HMXBs) with early type companion stars, and some of them contain accreting X-ray pulsars (Patel et al. 2004; Walter 2004; Lutovinov et al. 2005a). Remarkably these sources are confined in a relatively small region of the sky, close to the tangent to the
Norma Galactic spiral arm. Based on a previous study of the population of high mass X-ray binaries in our Galaxy (Grimm et al. 2002) it was proposed (Revnivtsev 2003) that such a concentration of absorbed HMXBs is the result of an enhanced star formation rate in this region and is an observational appearance of the Galactic spiral arm structure.

A similar enhanced population of HMXBs (more specifically, accreting X-ray pulsars) in the regions of spiral arms was emphasized by Koyama et al. (1990). Basing on the GINGA observations it was shown that in the region of the Scutum spiral arm tangent there exists a large number of accreting X-ray pulsars, likely variable, that was considered as an indication that they are in binary systems with $B[e]$ companion stars. A subsequent study of these systems with the ASCA observatory showed that many of them are absorbed (Sugizaki et al. 2001), and the comparison of the measured value $N_{\mathrm{H}}$ with the interstellar Galactic absorption indicates that the observed X-ray photoabsorption is likely intrinsic.

Previous studies of Galactic X-ray sources (e.g. Koyama et al. 1990; Negueruela 2004) suggest that sources with significant intrinsic photoabsorption in their spectra can be unambiguously classified as HMXBs, while the absence of large photoabsorption does not allow a reliable classification.

Such a difference in observational appearances of high mass and low mass X-ray binaries can be understood if we remember that in the majority of high mass X-ray binaries the compact objects accrete matter from the stellar wind of a young 
companion star, and therefore the dense stellar wind can create an envelope which will result in significant photoabsorption within the binary system. The best known examples of high mass X-ray binaries in our Galaxy are Cyg X-3, Vela X-1, GX301-2 and 4U1700-37. They all show the strong photoabsorption in their X-ray spectra.

In the case of low mass X-ray binaries (LMXBs) the main accretion goes through the inner Lagrangian point and therefore practically no obscuring matter is available in such systems (except for the rare class of dippers or accretion disk corona sources, see e.g. Kahn 1982). From this reasoning we can expect that any sample of HMXBs selected from soft X-ray data might lack systems with high intrinsic photoabsorption and therefore will lack a considerable number of HMXBs. In order to construct more uniform sample of HMXBs a hard $\mathrm{X}$-ray (>10-20 keV) energy band survey is important.

The international gamma ray observatory INTEGRAL (Winkler et al. 2003) is useful for the search of absorbed sources. The telescope IBIS/ISGRI (Ubertini et al. 2003, Lebrun et al. 2003) has a large effective area $(\sim 1000 \mathrm{sq} \mathrm{cm})$ and works in the hard X-ray energy range $(>20 \mathrm{keV})$, that largely excludes any influence of the photoabsorption of X-ray sources on the detection efficiency. It has a large field of view $\left(\sim 29^{\circ} \times 29^{\circ}\right)$ and good localization accuracy $\left(\sim 2-3^{\prime}\right)$.

The first two years of INTEGRAL observations provided more than 20 million seconds of data. The deepest hard X-ray ( 20-60 keV) surveys to date of the Galactic Center region - down to $\sim 1$ mCrab level (Revnivtsev et al. 2004), the Sagittarius Arm region - down to $\sim 1.5 \mathrm{mCrab}$ level (Molkov et al. 2004) and the Galactic plane - down to 2-3 mCrab level (Bird et al. 2004) were published. Here $1 \mathrm{mCrab}$ corresponds approximately to $\sim 1.4 \times 10^{-11} \mathrm{erg} \mathrm{s}^{-1} \mathrm{~cm}^{-2}$ for a source with a Crab-like spectrum. Much INTEGRAL data has became public and this opens the possibility to construct a highly sensitive flux limited sample of sources in the inner part of the Galaxy and try to make a systematic study of their broadband X-ray properties and their distribution in the Galaxy.

In this paper we focus on the analysis of the population of high mass X-ray binaries located in the Galactic plane from the Norma to Sagittarius spiral arms. This Galactic region was selected because it has the best statistics of the available INTEGRAL data. We plan to extend this study towards further Galactic spiral arms when more INTEGRAL data are available. We present the high quality broadband (1-100 keV) spectra of a number of newly discovered absorbed objects and report the discovery of X-ray pulsations from IGR J16465-4507.

\section{Data reduction}

For the reconstruction of the source broadband spectra we used all available X-ray data, which include data of the INTEGRAL observatory (all public data for revolutions up to 118 and the General Program data of the Galactic Center observations, Obs.ID 0120213 and 0220133) as well, data of RXTE, ASCA and XMM-Newton observatories. The data of the latter observatories were reduced using standard tools recommended by the Guest Observer Facilities (http://legacy.gsfc.nasa.gov). The data of all
INTEGRAL/IBIS/ISGRI observations were processed with the method described by Revnivtsev et al. (2004). In order to reconstruct the source spectra from IBIS/ISGRI data we used a ratio of fluxes measured in different energy channels to the fluxes measured by the ISGRI detector from the Crab nebula in the same energy bands. Detailed analysis of Crab nebula observations suggests that with the approach and software employed, a conservative estimation of uncertainty in measurements of absolute fluxes from the sources is about $10 \%$ and the shape of the spectrum is about 5\%. The last value was added to the following spectral analysis as a systematic uncertainty in each energy channel.

RXTE spectra of IGR J16318-4848 and IGR J16358-4726 were taken from the work of Revnivtsev (2003). In this work the special treatment was done in order to get rid of a strong contribution of the Galactic ridge emission.

\section{The sample and completeness}

The available INTEGRAL data now contain approximately $>20$ Ms of observations, in particular $>5 \mathrm{Ms}$ of the inner parts of the Galaxy $\left(|l|<90^{\circ}\right)$. In this paper we focus on the Galactic regions with Galactic latitudes $|b|<5^{\circ}$ and Galactic longitudes from $l>325^{\circ}$ to $l<50^{\circ}$ (from the Norma spiral arm tangent to the Sagittarius spiral arm tangent). These data allowed us to obtain the time averaged map of the region and to construct a flux limited sample of sources with a limiting sensitivity of $\sim 1.5 \mathrm{mCrab}$ in the $20-60 \mathrm{keV}$ energy band, that corresponds to an energy flux $\sim 1.8 \times 10^{-11} \mathrm{erg} \mathrm{s}^{-1} \mathrm{~cm}^{-2}$ for a Crab-like spectrum. This criterion was chosen to provide a maximal survey area with best possible sensitivity.

In the selected region of the Galactic plane $\left(325^{\circ}<l<50^{\circ}\right)$ INTEGRAL detects 89 different sources with a time average flux of $\gtrsim 1.5 \mathrm{mCrab}$ in the $20-60 \mathrm{keV}$ energy band. Among these sources there are 49 identified low mass X-ray binaries, 22 high mass X-ray binaries and 2 active galactic nuclei. Also there are supernova remnants and single pulsars. Ten sources remain unidentified. A detailed analysis of the available INTEGRAL sky map will be presented in a separate paper.

The list of high mass X-ray binaries presented in our sample is shown in Table 1. In this table we also present the value of the observed X-ray photoabsorption (Col. 3), the value of the interstellar Galactic $N_{\mathrm{H}}$ value inferred from radio maps (Dickey \& Lockman 1990), the average source flux in mCrab values and comments about the source nature.

Most of the presented HMXBs (16 of 23) are accretionpowered X-ray pulsars, one is a black hole candidate, 4U1700-377 is a well-known high mass X-ray binary without detectable pulsations. Other sources were included in the list because of the strong intrinsic absorption in their $\mathrm{X}$-ray spectra that is not typical for low mass X-ray binaries (IGR/XTE J17391-3021 was included based on the identification of the compaion star as a late O-type star, Smith 2004). One of the sources, IGR J16465-4507, was not detected in the INTEGRAL time averaged map, as it was discovered only in September 2004 (revolution 232, Lutovinov et al. 2004b). Follow-up observations with the XMM-Newton observatory revealed a strong absorption in the source 
Table 1. List of HMXB located in the Galactic plane from the Norma to Sagittarius spiral arms which were detected with INTEGRAL.

\begin{tabular}{|c|c|c|c|c|c|c|}
\hline Source & $\alpha(2000)$ & $\delta(2000)$ & $\begin{array}{c}N_{\mathrm{H}}, 10^{22} \mathrm{~cm}^{-2} \\
\text { Observed }\end{array}$ & $\begin{array}{c}N_{\mathrm{H}}, 10^{22} \mathrm{~cm}^{-2} \\
\text { Galactic }\end{array}$ & Flux, $\mathrm{mCrab}^{a}$ & Comments $^{b}$ \\
\hline 4U 1538-522 & 235.584 & -52.376 & $1.6[1]$ & 0.96 & $16.5 \pm 0.2$ & $\mathrm{P}$ \\
\hline AXJ161929-4945 & 244.871 & -49.758 & $14^{c}$ & 2.19 & $2.0 \pm 0.2$ & \\
\hline IGR J16318-4848 & 247.953 & -48.801 & $310^{c}$ & 2.07 & $21.8 \pm 0.2$ & \\
\hline AX J1631.9-4752 ${ }^{d}$ & 248.009 & -47.859 & $18^{c}$ & 2.13 & $13.0 \pm 0.2$ & $\mathrm{P}$ \\
\hline IGR J16358-4726 & 248.990 & -47.407 & $40^{c}$ & 2.20 & $3.11 \pm 0.21$ & $\mathrm{P}$ \\
\hline AX J163904-4642 & 249.757 & -46.676 & $58^{c}$ & 2.18 & $4.63 \pm 0.21$ & $\mathrm{P}$ \\
\hline IGR J16465-4507 & 251.648 & -45.118 & $72^{c}$ & 2.12 & $8.8 \pm 0.9$ & $\mathrm{P}$ \\
\hline IGR J16479-4514 & 252.032 & -45.206 & $12^{c}$ & 2.14 & $3.22 \pm 0.20$ & \\
\hline OAO 1657-415 & 255.199 & -41.653 & $40[2]$ & 1.76 & $68.2 \pm 0.2$ & $P$ \\
\hline 4U 1700-377 & 255.982 & -37.841 & $2-100[3]$ & 0.74 & $185.0 \pm 0.2$ & \\
\hline EXO 1722-363 & 261.286 & -36.280 & $50[4]$ & 1.50 & $7.18 \pm 0.13$ & $\mathrm{P}$ \\
\hline IGR/XTE J17391-3021 & 264.802 & -30.329 & $5-6[5]$ & 1.37 & $1.50 \pm 0.11$ & \\
\hline AX J1749.2-2725 & 267.335 & -27.511 & $10[6]$ & 1.62 & $1.51 \pm 0.11$ & $\mathrm{P}$ \\
\hline IGR/SAX J18027-2016 & 270.677 & -20.278 & $1-1.5[7]$ & 1.04 & $4.06 \pm 0.14$ & $\mathrm{P}$ \\
\hline AX J1820.5-1434 & 275.131 & -14.553 & $13[8]$ & 1.65 & $2.94 \pm 0.20$ & $\mathrm{P}$ \\
\hline AX J1838.0-0655 & 279.523 & -6.921 & $9^{c}$ & 1.86 & $2.36 \pm 0.24$ & \\
\hline GS $1843+00$ & 281.412 & 0.891 & $2.3[9]$ & 1.01 & $4.49 \pm 0.20$ & $\mathrm{P}$ \\
\hline XTE J1855-026 & 283.873 & -2.597 & $15[10]$ & 0.73 & $11.4 \pm 0.2$ & $\mathrm{P}$ \\
\hline $4 \mathrm{U} 1901+03$ & 285.914 & 3.215 & $7^{f}$ & 1.03 & $75.5 \pm 0.2$ & $\mathrm{P}$ \\
\hline 4U 1907+097 & 287.401 & 9.843 & $3-8[11]$ & 1.75 & $13.2 \pm 0.2$ & $\mathrm{P}$ \\
\hline $\mathrm{X} 1908+075$ & 287.699 & 7.598 & $10-50[12]$ & 1.48 & $13.4 \pm 0.2$ & $\mathrm{P}$ \\
\hline SS 433 & 287.950 & 4.990 & $-^{g}$ & 0.76 & $14.2 \pm 0.2$ & $\mathrm{BH}$ \\
\hline XTE J1858+034 & 284.693 & 3.429 & $6[13]$ & 1.89 & $15 \pm 1$ & $\mathrm{P}$ \\
\hline
\end{tabular}

${ }^{a}$ In the $20-60 \mathrm{keV}$ energy band.

${ }^{b}$ X-ray pulsar (P), black-hole candidate $(\mathrm{BH})$.

${ }^{c}$ Photoabsorption column obtained from our analysis of the broadband spectrum (see Table 2).

${ }^{d}$ The same as IGR J16320-4751.

${ }^{e}$ Source localization was improved in our analysis of the archival data of the ASCA observatory: RA $=16^{\mathrm{h}} 48^{\mathrm{m}} 07^{\mathrm{s}}$, Dec $=-45^{\circ} 12^{\prime} 21^{\prime \prime}($ an accuracy of $0.9^{\prime}$ ).

${ }^{f}$ Determined from the analysis of the public RXTE data.

${ }^{g}$ Central X-ray source is completely absorbed; the observed X-ray emission emerges from a hot outflowing jet (e.g. Watson et al. 1986).

References: [1] - Robba et al. (2001); [2] - Chakrabarty et al. (2002); [3] - Haberl \& Day (1992); [4] - Lutovinov et al. (2004a); [5] - Smith et al. (1998); [6] - Torii et al. (1998); [7] - Augello et al. (2003); [8] - Kinugasa et al. (1998); [9] - Piraino et al. (2000); [10] - Corbet et al. (1999); [11] - Roberts et al. (2001); [12] - Levine et al. (2004); [13] - Paul \& Rao (1998).

spectrum (see Table 1). Moreover, the improvement of the source position (Zurita \& Walter 2004) allowed observers to propose a blue supergiant star as its possible counterpart in the system (Smith 2004). The timing analysis of XMM-Newton data allowed us to detect pulsations of X-ray flux from this source (see below). Therefore we included IGR J16465-4507 in the list of HMXBs and have a sample of 23 sources in total.

We can estimate the number of extragalactic (mostly AGN) sources we should detect in the studied region with our sensitivity limit. For this purpose we can use either the fluxnumber function of Krivonos et al. (2005) in the energy band $20-60 \mathrm{keV}$ or use the more conventional flux-number function in the 2-10 keV energy band (e.g. Ueda et al. 2003; Sazonov \& Revnivtsev 2004) with the rescaled sensitivity limit (assuming, for example, a photon index of AGN spectra of $\Gamma \sim 1.7$ ). In both cases the estimated number of AGN in our region is of the order of 3-4 sources. Two AGN have already been identified - GRS 1734-292 (Pavlinsky et al. 1992) and IGR18027-1455 (Masetti et al. 2004).

\section{Absorbed sources}

The study of absorbed X-ray sources attracted much of attention with the launch of INTEGRAL (Matt \& Guainazzi 2003; Revnivtsev 2003; Rodriguez et al. 2003; Walter et al. 2003; Foschini et al. 2004; Combi et al. 2004, etc.). INTEGRAL is able to detect and measure the spectra of sources which are practically invisible for low energy X-ray instruments. The population of sources that demonstrates a high value of photoabsorption is not so small as to be disregarded - about 19 sources out of 23 , presented in Table 1 have a significant 


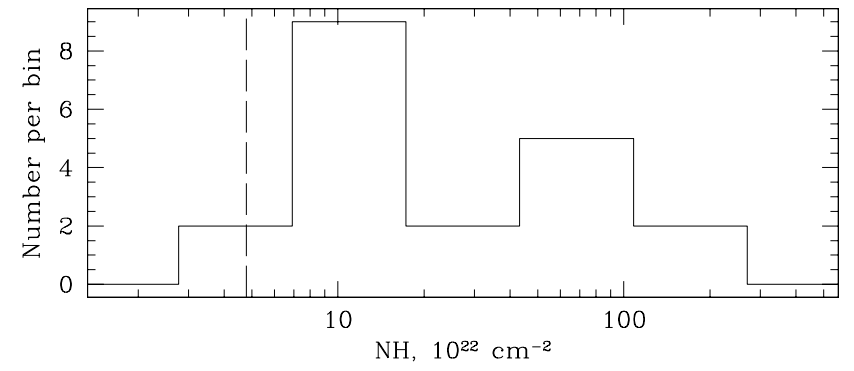

Fig. 1. Distribution of the absorption column value observed in X-ray spectra of HMXBs of our sample. The dashed line denotes the area where systematic uncertainties caused by high interstellar absorption in some Galactic regions prevents accurate measurements the source intrinsic photoabsorption value.

$\left(>5 \times 10^{22} \mathrm{~cm}^{-2}\right)$ intrinsic photoabsorption. In Fig. 1 we present the distribution of the observed $N_{\mathrm{H}}$ values in spectra of sources in our sample.

\subsection{Spectra}

To reconstruct the broadband spectra of absorbed sources we combine all available data from ASCA, RXTE, XMM-Newton and INTEGRAL observatories. The energy spectra of 8 sources in the $1-100 \mathrm{keV}$ energy band are presented in Fig. 2. These sources are the least studied in the broad energy band binaries located in the inner part of the Galaxy; most of them were discovered or rediscovered with INTEGRAL in hard $(>20 \mathrm{keV}) \mathrm{X}$-rays. Spectral points in a soft energy band $(<20 \mathrm{keV})$ obtained with non-simultaneous observations of ASCA, XMM-Newton and RXTE were renormalized to match the INTEGRAL/IBIS spectral normalization. Here we assumed that the shape of the source spectra is not variable and used time averaged data.

The obtained spectra can be well described by a simple powerlaw model modified by the cutoff at high energies and photoabsorption at soft X-rays that is typical for accreting neutron stars (see e.g. the spectrum of IGR/XTE J173913021 observed with INTEGRAL in the Galactic Center region, Lutovinov et al. 2005b). Best fit parameters are summarized in Table 2. Best fit models are shown in Fig. 2 by solid lines.

Note that for the source IGR J16465-4507 the spectrum is based on the data of XMM-Newton observations performed on Sept. 14, 2004 and the measurement of INTEGRAL (Lutovinov et al. 2004b). The solid line represents the spectral form typical of new absorbed sources - a power law with an exponential cutoff (this value was fixed at the energy $E_{\mathrm{c}}=30 \mathrm{keV}$ to better approximate the INTEGRAL point) and modified with the photoabsorption, determined with the help of XMM-Newton data.

It is seen that most of the sources have a somewhat similar spectral shape and parameters, in particular the folding (cutoff) energy. Only one source (AX J18380-0655) demonstrates a high energy cutoff value in its spectrum above $\sim 30 \mathrm{keV}$. Such high values of the cutoff are not typical for neutron star systems, and therefore this sources is likely a black hole
Table 2. Best-fit spectra parameters of 8 highly absorbed sources in the 1-100 keV energy band.

\begin{tabular}{lcccc}
\hline \hline Source & $\Gamma$ & $N_{\mathrm{H}}, 10^{22} \mathrm{~cm}^{-2}$ & $E_{c}, \mathrm{keV}$ & $\chi^{2} /$ (d.o.f.) \\
\hline AX J161929-4945 & $1.3 \pm 1.1$ & $14 \pm 4$ & $31 \pm 8$ & 0.64 \\
IGR J16318-4848 & $1.0 \pm 0.5$ & $310 \pm 70$ & $22 \pm 1$ & 0.95 \\
AX J1631.9-4752 & $0.7 \pm 0.2$ & $18 \pm 2$ & $13 \pm 1$ & 1.17 \\
IGR J16358-4726 & $0.7 \pm 0.5$ & $40 \pm 10$ & $16 \pm 5$ & 1.16 \\
AX J163904-4642 & $1.3 \pm 1.0$ & $58 \pm 11$ & $11 \pm 1$ & 0.57 \\
IGR J16465-4507 & $1.0 \pm 0.5$ & $72 \pm 6$ & 30 & 0.68 \\
IGR J16479-4514 & $1.4 \pm 0.8$ & $12 \pm 4$ & $32 \pm 2$ & 0.97 \\
AX J1838.0-0655 & $1.5 \pm 0.5$ & $9 \pm 3$ & $>50$ & 0.82 \\
\hline
\end{tabular}

candidate. All other systems demonstrate X-ray spectra typical of accreting neutron stars.

\subsection{Pulsations in IGR J16358-4726 and IGR J16465-4507}

For some of the newly discovered sources coherent pulsations of the X-ray flux were detected - IGR J16358-4726 (Patel et al. 2004), AX J163904-4642 (Walter 2004), AX J1631.94752 (Lutovinov et al. 2005a).

The available data of INTEGRAL and XMM-Newton observatories allowed us to confirm pulsations in the flux of IGR J16358-4726 and detect pulsations in the flux of IGR J16465-4507.

INTEGRAL observed IGR J16358-4726 during its outburst in spring of 2003 (Revnivtsev et al. 2003b,c). The source lightcurve in the $18-60 \mathrm{keV}$ energy band demonstrates clear pulse-like variations. The total exposure time of INTEGRAL observations used to construct the light curve of IGR J163584726 at the peak of its outburst was about $\sim 90 \mathrm{ks}$. The best period, obtained with the epoch-folding technique, is $P=5980 \pm$ $22 \mathrm{~s}$ (the error represents the $90 \%$ confidence interval; it was determined from the bootstrap Monte-Carlo simulations). The pulse profile of IGR J16358-4726 is presented in Fig. 3. As in the softer energies (see Patel et al. 2004) the source pulse profile consists of one peak. The pulse fraction in the $18-60 \mathrm{keV}$ energy band (which is defined as $P=\left(I_{\max }-I_{\min }\right) /\left(I_{\max }+I_{\min }\right)$, where $I_{\max }$ and $I_{\min }$ are intensities at the maximum and minimum of the pulse profile) is equal to $P=70 \pm 10 \%$, the same as in the CHANDRA energy band. The detailed timing analysis of the data of the XMM-Newton observations of IGR J164654507 allowed us to increase the number of known accreting $\mathrm{X}$-ray pulsars in the region of the Norma spiral arm.

The light curve of IGR J16465-4507 obtained with the XMM-Newton observatory during TOO observations of the source on Sept. 14, 2004 is shown in Fig. 4. Strong variations are presented in the light curve. The best fit period determined with the epoch-folding technique is $P=228 \pm 6 \mathrm{~s}$; the pulse fraction was estimated as $\sim 50-60 \%$. The folded light curve is shown in Fig. 4 (lower panel). Note that zero flux bins in the original light curve are caused by the Poissonian statistics. The mean count rate during the pulsation minimum is $\sim 0.03 \mathrm{cnts} / \mathrm{s}$, or $\sim 1 \mathrm{cnts}$ per bin $(40 \mathrm{~s})$. We could not search for pulsations in 

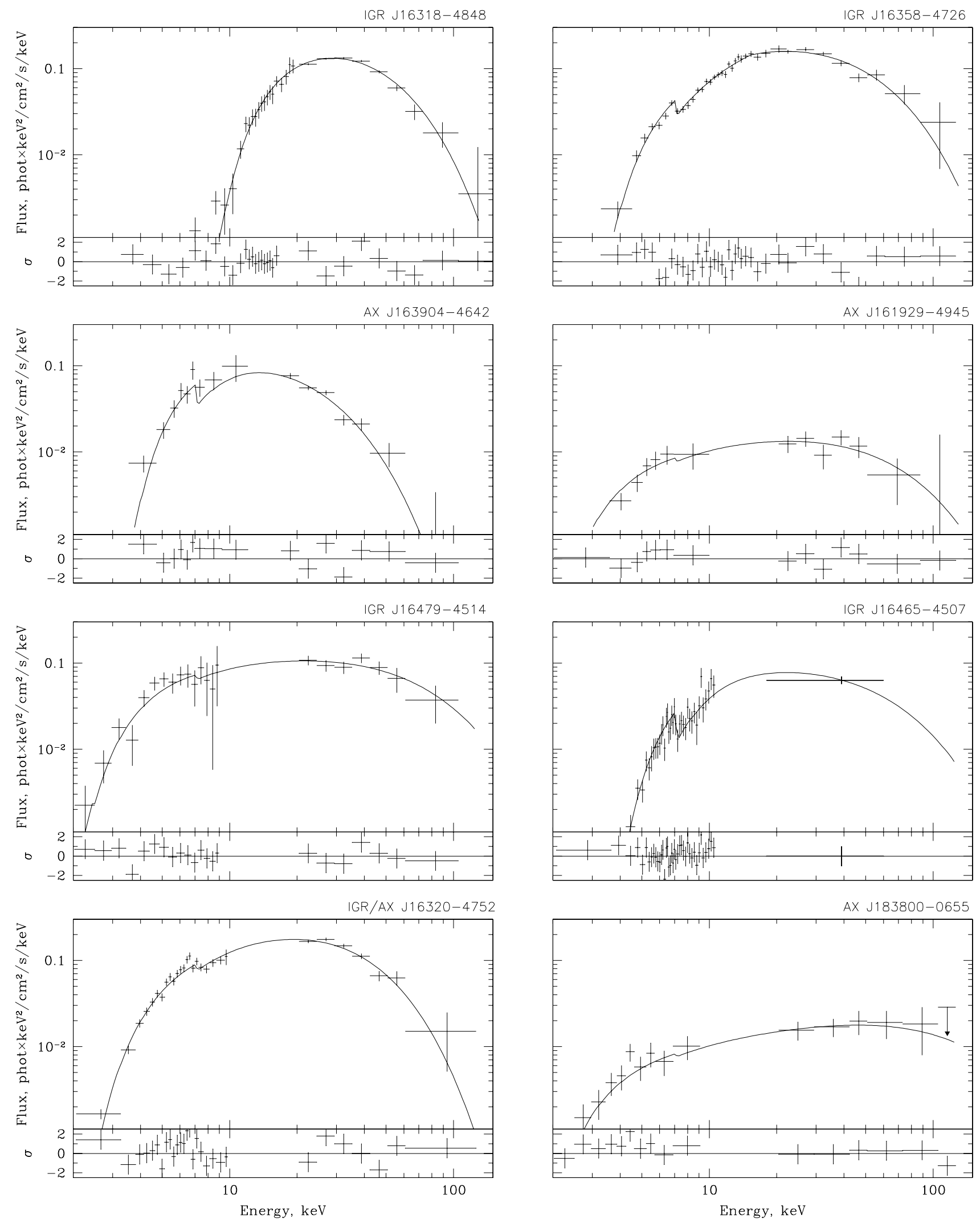

Fig. 2. Broadband energy spectra of 8 highly absorbed sources. Data in the hard energy part of spectra $(>20 \mathrm{keV})$ represent the INTEGRAL observatory measurements; data in standard X-rays were obtained by ASCA for AX J161929-4945, AX J1631.9-4752, AX J163904-4642, IGR J16479-4514, AX J1838.0-0655, RXTE for IGR J16318-4848, IGR J16358-4726 and XMM-Newton for IGR J16465-4507. Best fit models are shown by solid lines. Residuals are presented in the bottom panels. 


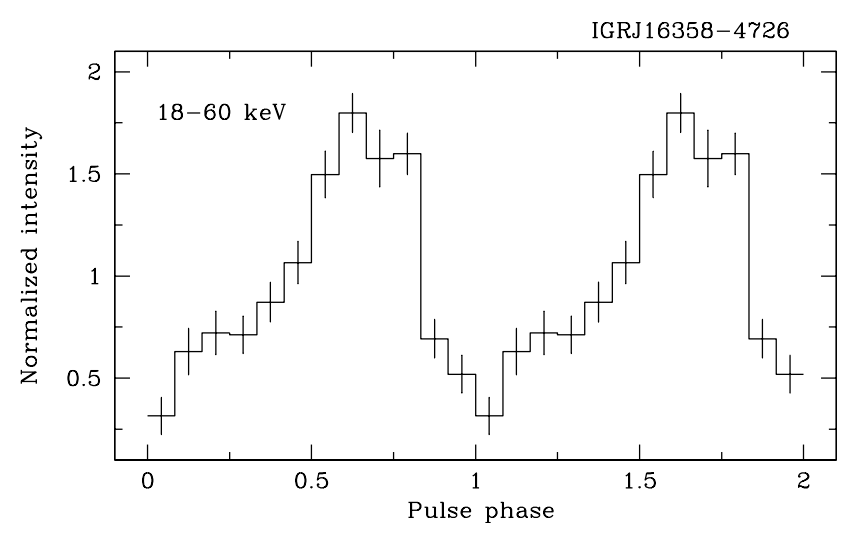

Fig. 3. Folded light curve of IGR J16358-4726 obtained with INTEGRAL/IBIS/ISGRI in the 18-60 keV energy band
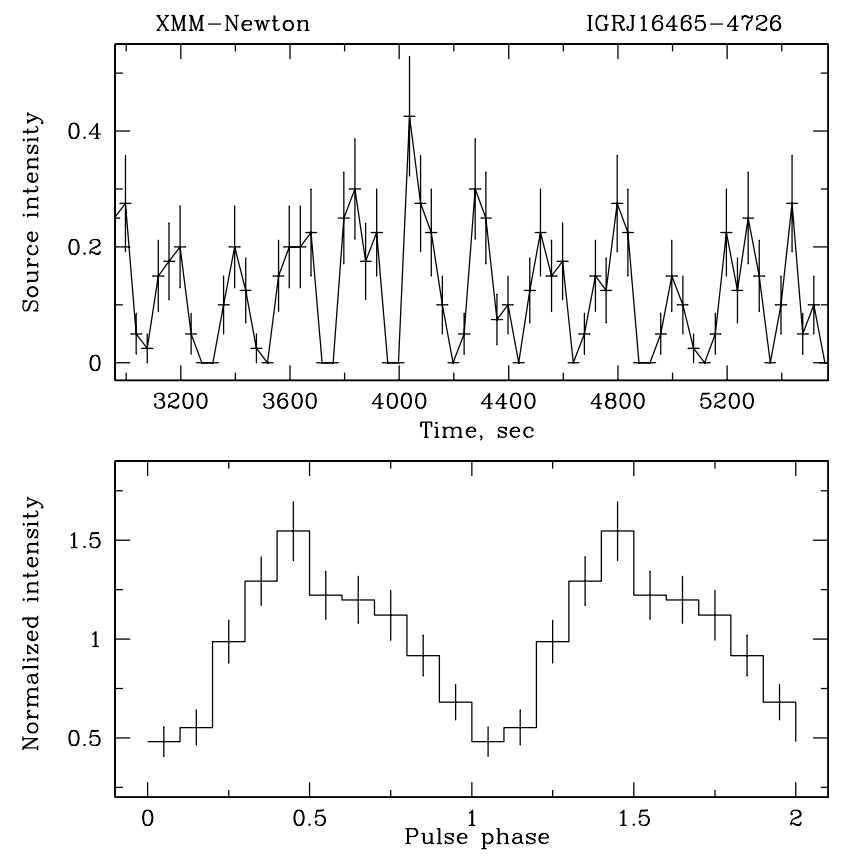

Fig. 4. (upper panel) Part of the light curve of IGR J16465-4507 obtained by XMM-Newton (the time bin is $40 \mathrm{~s}$ ). (bottom panel) Corresponding pulse profile of IGR J16465-4507 folded with the period of $228 \mathrm{~s}$.

the INTEGRAL data because they are currently not publically available.

For other sources no pulsations have been detected yet. However, similarities of their spectral shape to that of confirmed high mass X-ray binaries and the presence of the strong absorption in their spectra allow us to tentatively suggest that all they are HMXBs.

\section{Spatial distribution of the X-ray binaries}

The obtained sample of Galactic X-ray binaries allows us to study their spatial distribution in the comparison with the previous work of Grimm et al. (2002). In that work authors used the data of RXTE/ASM (energy band 1-12 keV) and that less sensitive to sources with high intrinsic photoabsorption. In addition to that, our limiting sensitivity is slightly better than that of Grimm et al. (2002). Assuming a spectrum

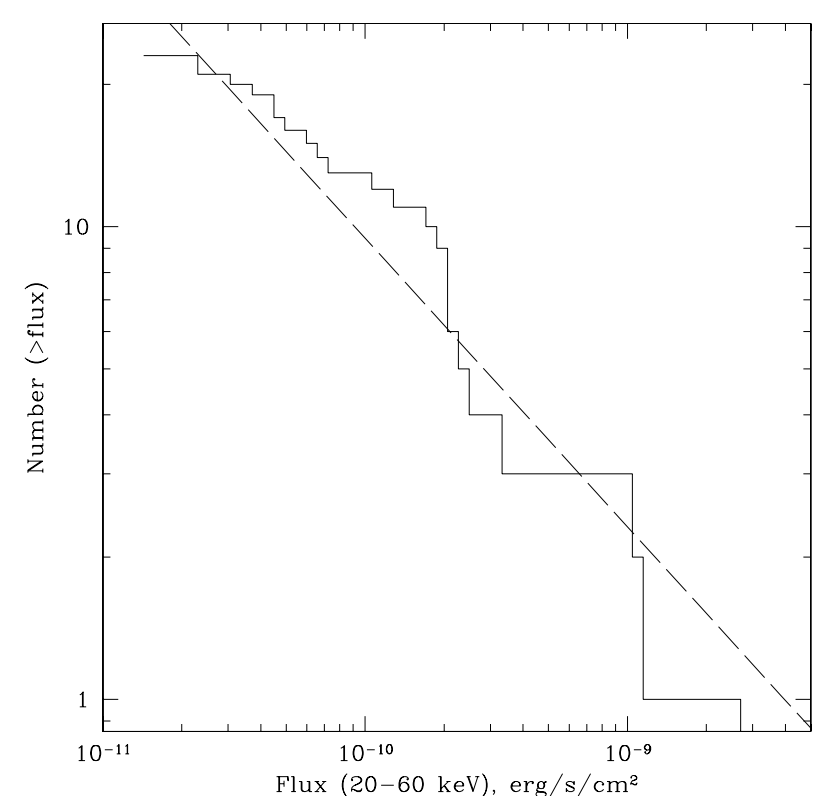

Fig. 5. Number-flux function of the Galactic HMXBs detected by INTEGRAL/IBIS/ISGRI in the inner part of the Galaxy. The dashed line represents the shape of the HMXB number-flux function obtained by Grimm et al. (2002).

of a typical accreting X-ray pulsar with zero intrinsic photoabsorption (power law with photon index $\Gamma=1.3$ with a high energy cutoff at $30 \mathrm{keV}$ ) our sensitivity of $\sim 1.8 \times$ $10^{-11} \mathrm{erg} \mathrm{s}^{-1} \mathrm{~cm}^{-2}$ in the $20-60 \mathrm{keV}$ energy band corresponds to $\sim 1-2 \times 10^{-11} \mathrm{erg} \mathrm{s}^{-1} \mathrm{~cm}^{-2}$ in the RXTE/ASM energy band $1-12 \mathrm{keV}$ (cf. $\sim 6 \times 10^{-11} \mathrm{erg} \mathrm{s}^{-1} \mathrm{~cm}^{-2}$ in Grimm et al. 2002).

In the region of our study $\left(325^{\circ}<l<50^{\circ}\right)$ we have detected in total 23 high mass X-ray binaries and HMXB candidates. This is more than 2 times larger than the sample used by Grimm et al. (2002) in the same region. In the region $l \sim 340^{\circ}$ our sample contains 6 sources, while Grimm et al. (2002) considered only one source. This is not a surprise because a significant part of our sample consists of new sources discovered by INTEGRAL which have strong photoabsorption. Note however, that the total sample of HMXBs used by Grimm et al. (2002) includes more than 50 HMXBs and is larger than ours.

The number-flux distribution of HMXB detected with INTEGRAL is presented in Fig. 5. The dashed line represents the number-flux relation obtained by Grimm et al. (2002).

The angular distribution of high mass X-ray binaries along the Galactic plane is presented by a histogram in Fig. 6 in the comparison with the distribution of LMXBs. To estimate the significance of the difference of the observed HMXB distribution from the LMXB one we used a Kolmogorov-Smirnov test in two ways: for the whole sample $\left(325^{\circ}<l<50^{\circ}\right)$ and for the first $\left(0^{\circ}<l<50^{\circ}\right)$ and fourth $\left(325^{\circ}<l<360^{\circ}\right)$ Galactic quadrants separately. We made this division in order to put the regions of interest in the middle of trial interval, where the K-S test is more sensitive. We build cumulative distributions for each of these cases and found that the probabilities that the HMXB distribution differs from LMXB one are $\sim 75 \%$ for the whole sample and $\sim 96 \%$ and $\sim 97 \%$ for first and fourth quadrants, respectively (the significance of the central peak for 


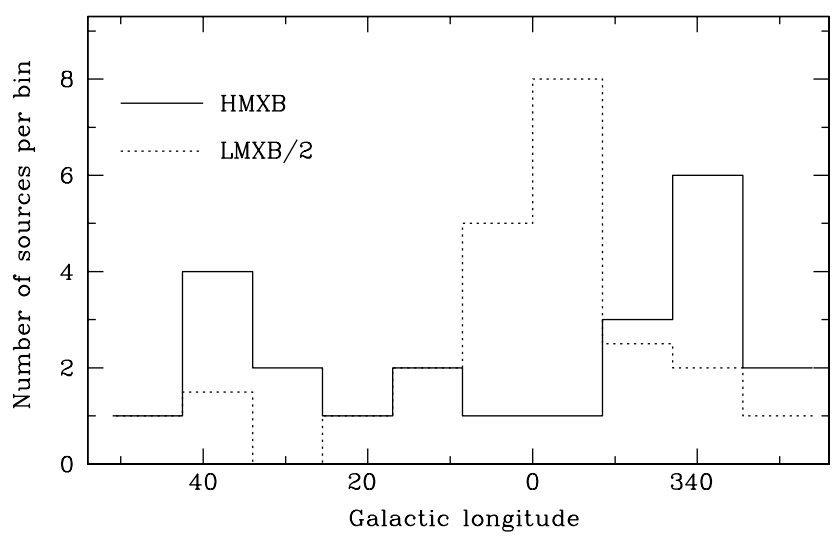

Fig. 6. Angular distribution of high mass X-ray binaries (HMXBs) and low mass X-ray binaries (LMXBs) in the inner Galaxy. The number of LMXB is devided by 2 .

the LMXB distribution is much higher, $>99.9 \%$ ). It is known (Grimm et al. 2002) that low mass X-ray binaries trace the stellar mass of the Galaxy and are concentrated in the Galactic center. From Fig. 6 and the K-S test it follows that the high mass $\mathrm{X}$-ray binary distribution is different to the LMXB one and not concentrated at the Galactic center. HMXBs, being the young population of X-ray sources in the Galaxy should be related to star formation regions (e.g. Galactic spiral arms) rather than to the region with high stellar mass density, and such a connection was indeed observed (e.g. Grimm et al. 2002, 2003).

In Fig. 7 we visualize the distribution of HMXB in the Galaxy with respect to the Galactic spiral structure. The number density of the sources is shown in gray scale. The spiral model of the Galaxy is based on optical and radio observations of HII regions (Taylor \& Cordes 1993). The dotted circles denote arcs of circles centered on the Sun and show maximal distances at which our sensitivity limit $(1.5 \mathrm{mCrab})$ allows us to detect $\mathrm{X}$-ray sources with the marked luminosities $\left(L_{\mathrm{x}}=10^{35} \mathrm{erg} / \mathrm{s}\right.$ and $\left.L_{\mathrm{x}}=10^{35.5} \mathrm{erg} / \mathrm{s}\right)$. The dashed lines denotes four logarithm spirals with pitch angle $12^{\circ}$ adopted by Vallee (1995). This model predicts the position of spiral arms in the opposite (with respect to the Galactic Center) part of the Galaxy, where measurements in the optical and radio bands are scarce, but these parts can be visible in X-rays.

The detailed comparison of the density of the high mass X-ray binary distribution with the spiral structure of the Galaxy (regions of the enhanced star formation) is not possible at the moment due to the limiting statistics of the sample used. This is the subject of a separate paper in which more INTEGRAL data for the whole Galaxy will be used. Here we only mention several possible reasons that may affect the observed HMXB density. They are: unknown distances to the sources and, as a sequence, their unknown exact positions; dynamics of the Galaxy and complicated motions of the Galactic spiral structure and stars from the moment of star formation to the X-ray source stage (e.g. a significant displacement of HMXBs with respect to the current position of the spiral arms was observed by CHANDRA in spiral galaxy M 83 Soria \& Wu 2003); the possible influence of previously unseen parts of the spiral arms, etc.

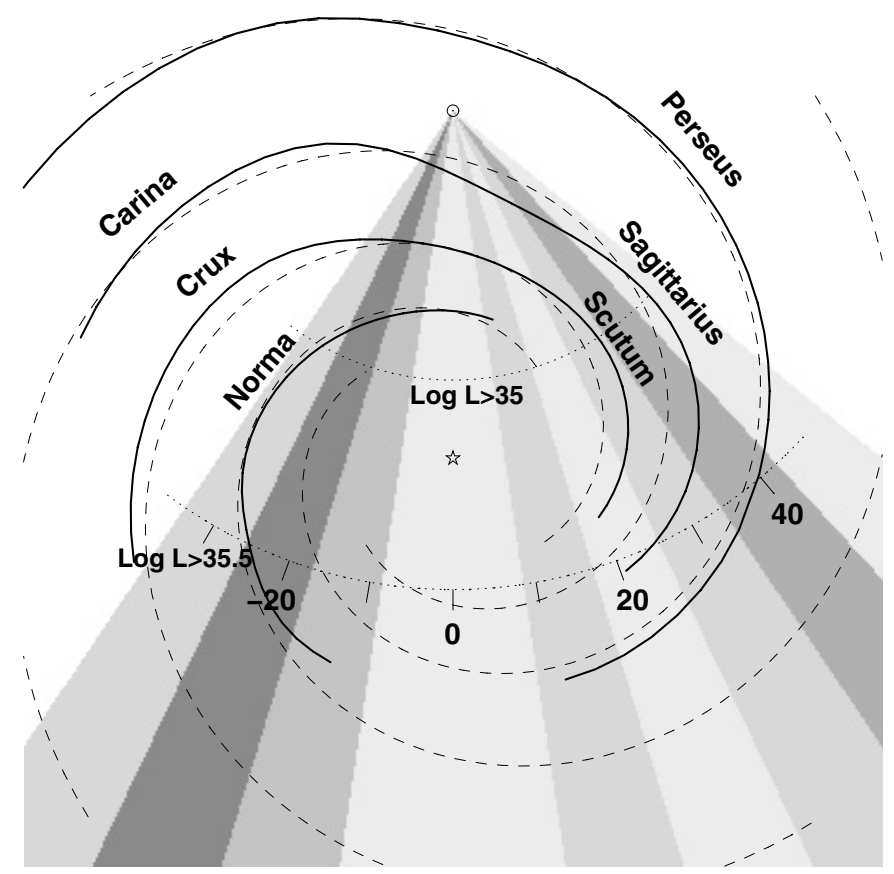

Fig. 7. Face-on view of the Galaxy with overlayed densities of HMXBs shown in gray scale (from 1 to 6, see Fig. 6). The distance of $8.5 \mathrm{kpc}$ of the Galactic Center from the Sun is assumed. The star denotes the position of the Galactic Center. The open circle with the dot denotes the position of the Sun. Solid lines show the position of the spiral arms by Taylor \& Cordes (1993), the dashed line shows logarithmic spiral arms by Vallee (1995), see also Russeil (2003).

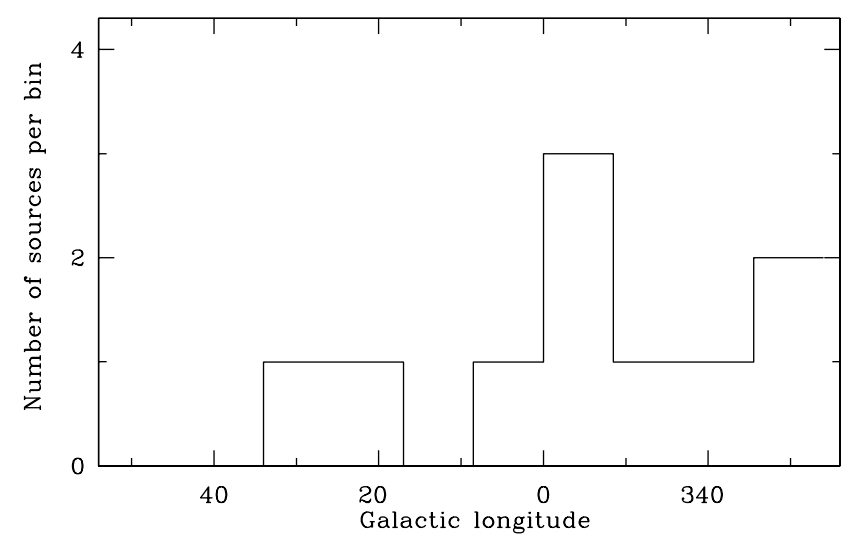

Fig. 8. The angular distribution of unidentified sources of our source sample along the Galactic plane.

\section{Unidentified sources}

In order to understand the possible influence of unidentified sources in our sample on the obtained distribution of HMXB we construct their distribution along the Galactic plane with the same coordinate binning as in Fig. 6.

This distribution (Fig. 8) shows that the unidentified sources cannot affect our conclusion about the distribution of high mass X-ray binaries in the inner part of the Galaxy. There is a small peak near the Galactic Center that may indicate that several of the unidentified sources are LMXBs. One or two of the unidentified sources may be AGN. 


\section{Summary}

- We presented a sample of high mass X-ray binaries in the inner part of the Galaxy from the Norma spiral arm region to the Sagittarius spiral arm region $\left(-35^{\circ}<l<50^{\circ}\right)$. This sample is significantly larger than the HMXBs sample in the same region of the sky, based on RXTE/ASM data, used by Grimm et al. (2002). The main reason for this is the hard $\mathrm{X}$-ray energy band (20-60 keV) used in the present observations which helped to reveal a considerable population of absorbed HMXBs.

- The majority of HMXB sources in our sample are significantly photoabsorbed.

- We performed for the first time a spectral analysis for 8 strongly absorbed sources of our sample. Based on the spectral approximations we argue that the majority of these sources should be neutron star binaries with a high mass companion. The exception is AX J183800-0655, which likely contains a black hole.

- We detected pulsations in the hard X-ray flux (18-60 keV) from IGR J16358-4726 with the period $P=5980 \pm 22 \mathrm{~s}$, thereby confirming the results of the CHANDRA observatory (Patel et al. 2004). In addition we found pulsations with the period $P=228 \pm 6 \mathrm{~s}$ in the $\mathrm{X}$-ray flux of IGR J16465-4507 using the data of XMM-Newton.

- We constructed the angular distribution of high mass X-ray binaries in the inner part of the Galaxy and showed that this distribution differs from the distribution of LMXBs, which are concentrated in the Galactic Center.

- A large fraction of the accretion powered X-ray pulsars in the Be-systems are transients. Therefore the continuation of INTEGRAL observations of the Galactic plane might lead to the discovery of many more similar systems and considerably increase the statistical significance of the observed displacement of the HMXB population with respect to the spiral arm tangents. Data of CHANDRA and XMM-Newton on other galaxies and detailed study of systems in our Galaxy might provide additional information about the motion of stars and spiral waves.

Acknowledgements. The authors thank Eugene Churazov for developing methods of analysis of the IBIS data and software. We would like to thank Sergei Sazonov for useful comments and the anonymous referee for critical review which allowed us to significantly improve the paper. This research has made use of data obtained through the INTEGRAL Science Data Center (ISDC), Versoix, Switzerland, Russian INTEGRAL Science Data Center (RSDC), Moscow, Russia and High Energy Astrophysics Science Archive Research Center Online Service, provided by the NASA/Goddard Space Flight Center. This work was partially supported by the program of Russian Academy of Sciences "Non-stationary phenomena in astronomy". A.L., M.R., P.S. and S.M. acknowledge the support of RFFI grant 04-02-17276.

\section{References}

Augello, G., Iaria, R., Robba, N. R., et al. 2003, ApJ, 596, L63 Bird, A., Barlow, E., Bassani, L., et al. 2004, ApJ, 607, L33
Chakrabarty, D., Wang, Z., Juett, A. M., Lee, J. C., \& Roche, P. 2002, ApJ, 573, 789

Combi, J., Ribo, M., Mirabel, F., \& Sugizaki, M. 2004, A\&A, 422, 1031

Corbet, R. H. D., Marshall, F. E., Peele, A. G., \& Takeshima, T. 1999, ApJ, 517, 956

Courvoisier, T., Walter, R., Rodriguez, J., et al. 2003, IAUC, 8063

de Plaa, J., Hartog, P., Kaastra, J., et al. 2003, Astron. Telegram, 119, 1

Dickey, \& Lockman 1990, Ann. Rev. Ast. Astr. 28, 215

Foschini, L., Tomsick, J., Rodriguez, J., et al. 2004, Proc. of the 5th INTEGRAL workshop, The INTEGRAL Universe, ed. V. Schönfelder, G. Lichti, \& C. Winkler (ESA Publications Division), SP-552, 247

Filliatre, P., \& Chaty, S. 2004, ApJ, 616, 469

Grimm, H.-J., Gilfanov, M., \& Sunyaev, R. 2002, A\&A, 391, 923

Grimm, H.-J., Gilfanov, M., \& Sunyaev, R. 2003, MNRAS, 339, 793

Haberl, F., \& Day, C. S. R. 1992, A\&A, 263, 241

Kahn S. 1982, Adv. Space Res., 2, 85

Kinugasa, K., Torii, K., Hashimoto, Y., et al. 1998, ApJ, 495, 435

Koyama, K., Kawada, M., Kunieda, H., Tawara, Y., \& Takeuchi, Y. 1990, Nature, 343, 148

Krivonos, R., Vikhlinin, A., Churazov, E., et al. 2005, ApJ, 625, 89

Lebrun, F., Leray, J. P., Lavocat, P., et al. 2003, A\&A, 411, L141

Levine, A., Rappaport, S., Remillard, R., \& Savcheva, A. 2004, ApJ, 617,1284

Lin, C. C., Yuan, C., \& Shu, F. H. 1969, ApJ, 155, 721

Lutovinov, A., Tsygankov, S., Revnivtsev, M., et al. 2004a, Proc. of the 5th INTEGRAL workshop, The INTEGRAL Universe, ed. V. Schönfelder, G. Lichti, \& C. Winkler (ESA Publications Division), SP-552, 253

Lutovinov, A., Rodriguez, J., Budtz-Jorgensen, C., et al. 2004b, Astron. Telegram, 329, 1

Lutovinov, A., Rodriguez, J., Revnivtsev, M., \& Shtykovskiy, P. 2005a, A\&A, 433, L41

Lutovinov, A., Revnivtsev, M., Molkov, S., \& Sunyaev, R. 2005b, A\&A, 430, 997

Matt, G., \& Guainazzi, M. 2003, MNRAS, 341, L13

Molkov, S., Cherepashchuk, A., Lutovinov, A., et al. 2004, Astron. Letters, 30, 534

Masetti, N., Palazzi, E., Bassani, L., et al. 2004, A\&A, 426, L41

Murakami, H., Dotani, T., \& Wijnands, R. 2003, IAUC, 8070

Negueruela, I. 2004, Proc. of JENAM-2004, in press [arXiv: astro-ph/0411759]

Patel, S., Kouveliotou, C., Tennant, A., et al. 2004, ApJ, 602, L45

Paul, B., \& Rao, A. R. 1998, A\&A, 337, 815

Pavlinsky, M., Grebenev, S., \& Sunyaev, R. 1992, Astron. Lett., 18, 88

Piraino, S., Santangelo, A., Segreto, A., et al. 2000, A\&A, 357, 501

Revnivtsev M. 2003, Astron. Lett., 29, 644

Revnivtsev, M., Sazonov, S., Gilfanov, M., et al. 2003a, Astron. Lett., 29, 587

Revnivtsev, M., Tuerler, M., Del Santo, M., et al. 2003b, IAUC, 8097

Revnivtsev, M., Lutovinov, A., \& Ebisawa, K. 2003c, Astron. Telegram, 131, 1

Revnivtsev, M., Sunyaev, R., Varshalovich, D., et al. 2004, Astr. Lett., 30,382

Robba, N. R., Burderi, L., Di Salvo, T., Iaria, R., \& Cusumano, G. 2001, ApJ, 562, 950

Roberts, M. S. E., Michelson, P. F., Leahy, D. A., et al. 2001, ApJ, 555,967

Rodriguez, J., Tomsick, J., Foschini, L., et al. 2003, A\&A, 407, L41

Sazonov, S. Y., \& Revnivtsev, M. G. 2004, A\&A, 423, 469 
Smith, D. M., Main, D., Marshall, F., et al. 1998, ApJ, 501, L181 Smith, D. 2004, Astron. Telegram, 338, 1

Soria, R., \& Wu, K. 2003, A\&A, 410, 53

Sugizaki, M., Mitsuda, K., Kaneda, H., et al. 2001, ApJS, 134, 77

Tawara, Y., Yamauchi, S., Awaki, H., et al. 1989, PASJ, 41, 473

Taylor, J. H., \& Cordes, J. M. 1993, ApJ, 411, 674

Tomsick, J., Lingenfelter, R., Corbel, C., et al. 2004, Proc. of the 5th INTEGRAL workshop, The INTEGRAL Universe, ed. V. Schönfelder, G. Lichti, \& C. Winkler (ESA Publications Division), SP-552, 413

Torii, K., Kinugasa, K., Katayama, K., et al. 1998, ApJ, 508, 854
Ubertini, P., Lebrun, F., Di Cocco, G., et al. 2003 A\&A, 411, L131

Ueda, Y., Akiyama, M., Ohta, K., \& Miyaji, T. 2003, ApJ, 598, 886 Vallee, J. P. 1995, ApJ, 454, 119

Walter, R., Rodriguez, J., Foschini, L., et al. 2003, A\&A, 411, L427

Walter, R., \& INTEGRAL Survey Team 2004, AAS/High Energy Astrophysics Division, 8,

Watson, M. G., Stewart, G. C., King, A. R., \& Brinkmann, W. 1986, MNRAS, 222, 261

Winkler, C., Courvoisier, T., Di Cocco, G., et al. 2003 A\&A, 411, L1

Zurita, H., \& Walter, R. 2004, Astron. Telegram, 336, 1 